\title{
Non-interactive multiple predator effects on tadpole survival
}

Ramos Real, O J ; Van Buskirk, J

\begin{abstract}
We estimated interactions among and within three species of predator in their effects on prey survival using short-term predation experiments. The prey were Rana temporaria tadpoles, and the predators were dragonfly larvae (Anax imperator), newts (Triturus alpestris), and backswimmers (Notonecta glauca). Mortality rate per predator imposed by Triturus and Notonecta did not decline with predator density, whereas the predation rate of Anax was strongly reduced when the number of predator individuals increased. Impacts of all three predators were not altered by the presence of other species in pairwise combinations. This system is therefore characterized by interference between individual dragonflies but relatively independent effects of predator species. These results were largely predictable based on the natural history of the predators, and are encouraging for attempts to model communities as assemblages of interacting species.
\end{abstract}

DOI: https://doi.org/10.1007/s00442-011-2208-5

Posted at the Zurich Open Repository and Archive, University of Zurich

ZORA URL: https://doi.org/10.5167/uzh-64498

Journal Article

Published Version

Originally published at:

Ramos Real, O J; Van Buskirk, J (2012). Non-interactive multiple predator effects on tadpole survival. Oecologia, 169(2):535-539.

DOI: https://doi.org/10.1007/s00442-011-2208-5 


\title{
Non-interactive multiple predator effects on tadpole survival
}

\author{
Oscar Ramos • Josh Van Buskirk
}

Received: 16 May 2011 / Accepted: 14 November 2011 / Published online: 8 December 2011

(C) Springer-Verlag 2011

\begin{abstract}
Interactions among and within three species of predators were estimated in terms of their effects on prey survival using short-term predation experiments. The prey were tadpoles (Rana temporaria), and the predators were dragonfly larvae (Anax imperator), newts (Triturus alpestris), and backswimmers (Notonecta glauca). Mortality rate per predator imposed by Triturus and Notonecta did not decline with predator density, whereas the predation rate of Anax was strongly reduced when the number of predator individuals increased. Impacts of all three predators were not altered by the presence of other species in pairwise combinations. This system is therefore characterized by interference between individual dragonflies but relatively independent effects of predator species. These results were largely predictable based on the natural history of the predators and are encouraging for attempts to model communities as assemblages of interacting species.
\end{abstract}

Keywords Multiple predator species - Additive effects . Emergent effects $\cdot$ Density dependence

\section{Introduction}

Predation is among the most important factors influencing the structure and function of ecological systems (Sih et al. 1985; Lima 2002). In nature, multiple predator species are

Communicated by Ross Alford.

O. Ramos $(\bowtie) \cdot$ J. Van Buskirk

Institute of Evolutionary Biology and Environmental Studies,

University of Zürich, Winterthurerstrasse 190,

8057 Zurich, Switzerland

e-mail: o.j.ramos.real@gmail.com often present together and may differ in their effects on the abundance, diversity, and phenotypes of prey (Wilbur and Fauth 1990; Sih et al. 1998) and on the functional properties of ecosystems (Ives et al. 2005; Casula et al. 2006). Multiple predator impacts may be understood by the extent to which the predators interact. On the one hand, individual predators of the same or different species may act as functional units with independent effects on lower levels in the food web; on the other hand, individual predators may influence one another's foraging rate on prey by means of direct or indirect interactions. These alternatives have quite different implications for theoretical modeling. If predators act independently, their impacts can be predicted by combining individuals or species additively in community models. However, the second case is more complex because mortality rates of prey must be modeled as a function of the density and composition of predators.

Non-independent effects of multiple predator species, termed emergent multiple predator effects (MPEs; Sih et al. 1998), have been reported in many predator-prey systems (reviewed in Sih et al. 1998; Schmitz 2007). Factors causing emergent MPEs include competitive interference, intraguild predation among the predators, and interaction modifications (i.e., indirect effects of predator or prey phenotypic changes; Sih et al. 1998). These factors can also influence interactions among conspecific predators foraging together (Vance-Chalcraft et al. 2004; Griffen 2006). Investigations of multiple predator systems should be designed to detect non-independence at both intra- and inter-specific levels.

The null model in studies of MPEs is that the predators have independent effects. Two different experimental designs, termed additive and substitutive, have been used to detect emergent MPEs (Griffen 2006; Schmitz 2007). The more common additive design evaluates the net impact on 
prey as additional predators are added to the system; this approach determines whether the effect of one predator depends on the presence of another (Billick and Case 1994). Species composition and density covary under the additive design, so it is impossible to identify which of the two causes non-independence. The substitutive design (also termed a replacement series) measures prey survival while one predator species is substituted for another, with predator density held constant (Jolliffe 2000). This approach asks whether the two predators have equivalent impacts on prey, essentially calibrating the effects of one species against the other. Rather few studies of multiple predator systems have deployed both designs, although both in combination are needed to evaluate the impacts of both species composition and density of predators (Vance-Chalcraft and Soluk 2005; Griffen 2006; Carey and Wahl 2010).

Here we report a study of multi-predator effects involving three co-occurring species of aquatic predators and their tadpole prey. The experiment included both additive and substitutive designs, with the aim of evaluating whether the impact of a predator is affected by the presence of another species or another individual of the same species and whether the three predators have equivalent impacts. Thus, for each predator species we addressed two specific questions: (1) are there non-linear effects on predation rates of increasing conspecific density? and (2) does the presence of a second predator species create emergent MPEs?

We utilized predator species with distinct microhabitats and hunting strategies because this approach enabled us to make specific predictions about our results. Emergent MPEs are expected to occur when predators have overlapping habitat domains and similar foraging modes (Sih et al. 1998; Schmitz 2007). The underlying rationale for this is that closely interacting predators have many opportunities for distraction, interference, and cannibalism or intra-guild predation and, consequently, they are less effective at capturing prey when together. In our study, this situation is fulfilled in treatments with increasing densities of the same species; we therefore predict non-independent impacts of conspecific predators. However, independent impacts on prey risk are expected in our experiment when different predators occur together because the three species are ecologically distinct. Adult Notonecta glauca backswimmers (Hemiptera: Notonectidae) are active, visual foragers in the open water, descending onto their prey from above or taking prey from the surface (Sih 1982; Streams 1987). Larval Anax imperator dragonflies (Odonata: Aeshnidae) are sitand-wait predators of vegetated and benthic microhabitats (Corbet 1957; Folsom and Collins 1984). Foraging in aquatic adult Triturus alpestris newts (Caudata: Salamandridae) occurs mostly along the bottom of the pond (Griffiths 1996). These differences in zone of occurrence and method of hunting decrease the likelihood that these species will interact strongly when paired together. The prey in our experiment, larvae of the European common frog (Rana temporaria), have relatively broad microhabitat distributions that overlap those of all three predators; this should reduce the likelihood of emergent MPEs (Schmitz 2007). Thus, we made two clear predictions: conspecific predators should interact nonlinearly, whereas heterospecific combinations of predators should have independent effects.

\section{Methods}

We exposed tadpoles of $R$. temporaria in short-term predation trials to treatments that varied in the type of predator and the number of individual predators foraging together. The design included the three pairwise systems obtained from combining larval A. imperator odonates, adult T. alpestris newts, and adult N. glauca backswimmers. Each combination of predator species comprised a $2 \times 2$ factorial design with the presence and absence of one individual of each predator species, along with two additional treatments containing two individual conspecific predators (Fig. 1). There were 40 replicates of the predator treatments and 24 replicates of the predator-free treatment, distributed evenly among eight 1-day trials between 14 April and 7 May 2009. Experimental units were plastic tubs $\left(0.28 \mathrm{~m}^{2}\right.$, $80 \mathrm{~L}$ ) located outdoors in a field on the University of Zurich campus. Habitat structure was provided in the form of two packets of plastic ribbons, one floating and the other held against the bottom, each with four ribbons measuring $40 \times 4 \mathrm{~cm}$. The ribbons covered about $45 \%$ of the surface area of the tub. We filled tubs at 0800 hours on the day before the trial, and added 12 tadpoles $(60-80 \mathrm{mg})$ to each tub at 1000 hours. Predators were introduced the following morning at 0800 hours, and survivors were counted $9 \mathrm{~h}$ later at 1700 hours. The duration of the trials prevented tadpoles from developing induced defenses-beyond the immediate behavioral response-and ensured that roughly half the prey remained alive at the end. A new set of animals was used in each trial. Although the tubs were smaller than most wetlands occupied by these species in nature, the densities used here (43 tadpoles $/ \mathrm{m}^{2}, 0-7.1$ predators $/ \mathrm{m}^{2}$ ) were well within the range of natural densities observed locally (Van Buskirk 2005).

\section{Statistical analysis}

Tadpole mortality rate was calculated under a negative exponential model, assuming constant mortality risk through time: $N_{\mathrm{t}}=N_{0} \times \exp (-m t)$, where $N_{\mathrm{t}}$ is the number alive at the end of the trial, $N_{0}$ is the number at the beginning (12 tadpoles), $m$ is the per capita mortality rate per 


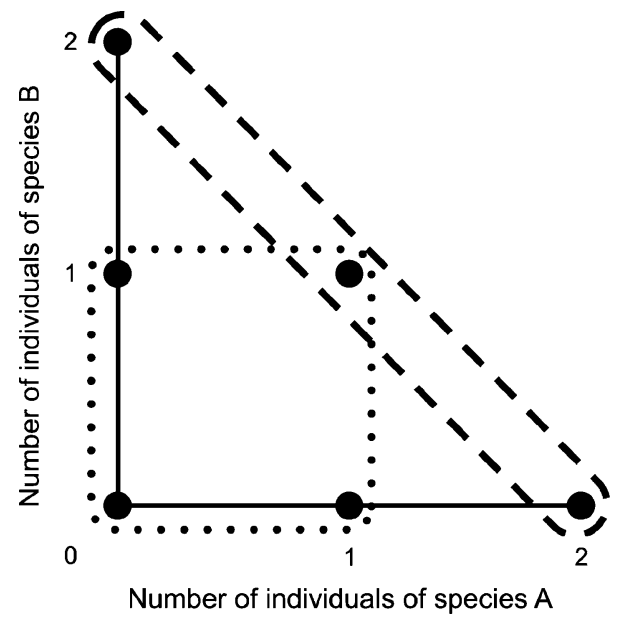

Fig. 1 Design of the experiment to evaluate emergent multiple predator effects on mortality of Rana temporaria tadpoles caused by three kinds of predators (Anax imperator larvae, adult Triturus alpestris, and adult Notonecta glauca). The experimental design was repeated for each pairwise combination. $A$ and $B$ represent the two predator species and black circles are treatments that were included. The dotted line illustrates the additive design and the dashed line the substitutive design

hour, and $t$ is the elapsed time $(9 \mathrm{~h})$. This is formally equivalent to analyzing log-transformed data, as recommended elsewhere (Billick and Case 1994). The slopes of negative exponential mortality curves combine additively (i.e., linearly) when there are no emergent MPEs and predator impacts on mortality are independent. There was variation in mortality among days $\left(F_{7,376}=6.63, P<0.001\right)$, so we performed analyses on residuals after correcting for day. The grand mean was added back to produce Fig. 2.

For each predator species, we determined whether the increase in intra-specific density caused nonlinear effects on tadpole mortality by comparing three alternative models. The null model (including only the intercept) would be supported if predators did not affect tadpole mortality rate; the linear model would be supported if conspecific predators had independent linear effects on mortality (i.e., two predators created twice the instantaneous risk as a single predator); the second order model including the square of predator density would be supported if the second predator resulted in more or less than a doubling in mortality rate (i.e., an emergent MPE). We compared Akaike weights from the small-sample version of Akaike's information criterion (AICc) to identify the best-supported model from among these three (Burnham and Anderson 2002).

In each pairwise predator system, we tested for emergent MPEs using both the additive and substitutive designs (Fig. 1). Under the additive approach, an interaction between predators was identified by a significant interaction term in a two-way analysis of variance (ANOVA) including the four treatments enclosed within the dotted

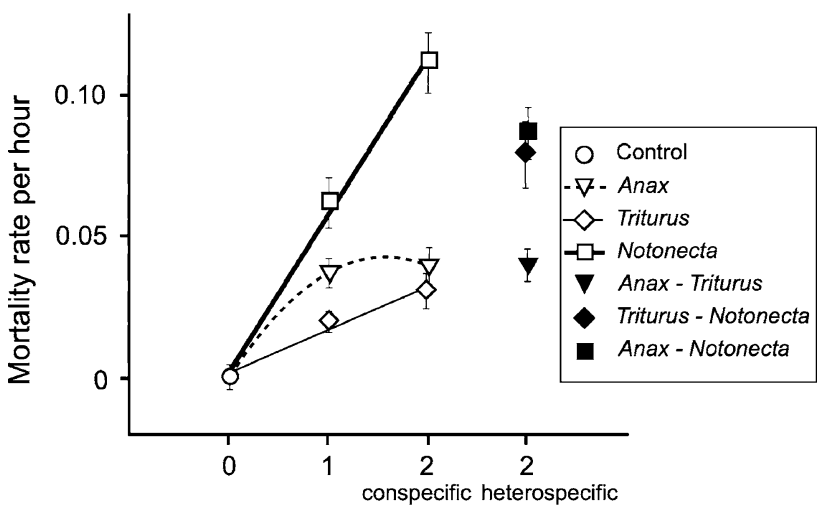

Number of predators

Fig. 2 The mortality rates of $R$. temporaria tadpoles when exposed to different numbers and combinations of predators: larval A. imperator, adult $T$. alpestris, and adult $N$. glauca (mean hourly rate as defined in the text \pm 1 standard error). Open circle Predator-free control (24 replicates), other open symbols single-species predator treatments (40 replicates), filled symbols three heterospecific predator treatments (40 replicates). Lines represent the fitted model with the highest Akaike information criterion weight. Overlapping symbols on the horizontal axis are offset intentionally for illustrative purposes

line in Fig. 1 (Billick and Case 1994). The substitutive approach asked whether the mortality rate imposed by the predators together differed from the mean of mortality caused by each predator species when alone, at the same total density (Jolliffe 2000; Griffen 2006). This was done using ANOVA on mortality in the three treatments enclosed by the dashed line in Fig. 1 followed by a planned contrast comparing the predator combination with the midpoint of the two pure-species treatments. Analyses were performed in R 2.9.1.

\section{Results}

Of the three predators, Notonecta imposed the highest mortality rate on the relatively small tadpoles used in this experiment (Fig. 2). The model best supported by the data for Notonecta and Triturus was the linear relationship between mortality and predator density (Akaike weights $w 0.72$ and 0.70 ). The quadratic model was best supported for Anax ( $w=0.83$ ) because two dragonflies together in the tub killed tadpoles at about the same combined rate as a single dragonfly alone (Fig. 2). Thus, individual Anax were only half as dangerous when they occurred in pairs. The null model was in all cases poorly supported $(w<0.01)$.

Tadpole mortality in the two-predator combinations did not differ significantly from expectations based on the additive or substitutive approaches. Under the additive model, the two-way interactions were non-significant for all three predator combinations (all $P$ values $>0.13$ ). Under the substitutive approach, contrasts comparing the two predators 
Table 1 Analyses of variance on the three treatments encircled by the dashed line in Fig. 1, for all pairwise combinations of predator species (Anax imperator, Triturus alpestris, and Notonecta glauca)

\begin{tabular}{lrlll}
\hline Source & $d f$ & \multicolumn{1}{l}{ SS } & Test statistic & $P$ \\
\hline $\begin{array}{l}\text { Anax-Triturus } \\
\quad \text { Predator }\end{array}$ & 2 & 0.002 & 0.607 & 0.547 \\
$\begin{array}{l}\text { Residual } \\
\text { Contrast }\end{array}$ & 117 & 0.205 & & \\
$\begin{array}{l}\text { Anax-Notonecta } \\
\text { Predator }\end{array}$ & 2 & 0.105 & 12.21 & $<0.001$ \\
$\begin{array}{l}\text { Residual } \\
\text { Contrast }\end{array}$ & 117 & 0.502 & & \\
$\begin{array}{l}\text { Triturus-Notonecta } \\
\quad \text { Predator }\end{array}$ & 2 & 0.137 & 19.69 & 0.780 \\
$\begin{array}{l}\text { Residual } \\
\text { Contrast }\end{array}$ & 117 & 0.408 & & \\
\hline
\end{tabular}

The planned contrasts compared the mortality rate observed in the presence of two individual predators of different species with that predicted from treatments having two predators of the same species. The contrast was not performed for the Anax-Triturus combination because there was no significant variation among treatments. Test statistics were the $F$ value for the analysis of variance (ANOVA) and $t$ value for contrasts

together with the midpoint of the two pure-predator treatments were not significant for the Anax-Notonecta and Triturus-Notonecta combinations (Table 1). This is clearly visible in Fig. 2: mortality rate under the mixed-species combinations lies almost exactly halfway between mortality when the two individual predators were of the same species. We did not perform this contrast for the Anax-Triturus combination because there was no significant variation among the three predator treatments (Table 1).

\section{Discussion}

The three predator species used in this study did not produce emergent MPEs on the survival of $R$. temporaria tadpoles. The tadpole mortality rate under combinations of predators was accurately predicted from information on mortality in single-predator situations. This result suggests that foraging effort and hunting behavior of the predators were not strongly affected by the presence of another predator. The same conclusion applies to intra-specific interactions for Triturus and Notonecta, but not for Anax. Instantaneous risk imposed by the first two species increased linearly with the number of individual predators, whereas two Anax larvae consumed no more tadpoles than a single Anax foraging alone. Thus, independent effects were prevalent in the predator systems evaluated here, except for density-dependence in dragonfly larvae. These results are largely consistent with expectations based on the natural history of these three predator species.

Additive and substitutive experimental designs address two slightly different hypotheses on interactions among predators. Testing for an interaction within the additive design asks whether the effect of the two-species combination can be predicted from the effects of single individuals of the two species in isolation (Billick and Case 1994). Although density and diversity are confounded, the test determines whether the predators interact at all. The substitutive approach estimates whether the impact of an individual is altered when a conspecific is replaced by a predator of another species, essentially comparing intra-specific and inter-specific interference. In our study, the results from both approaches were in agreement that the extent of interaction among predator species is minimal. This is unusual; most previous studies that employed both designs obtained different answers from the two approaches (Vance-Chalcraft and Soluk 2005; Griffen 2006; Carey and Wahl 2010; and see re-examined data in Schmitz 2007).

The natural history of the three predators, reviewed in the "Introduction", supports prevailing hypotheses about conditions under which emergent MPEs occur (Sih et al. 1998; Schmitz 2007). Multi-predator impacts on prey are predicted to be substitutable when spatial habitat domains (sensu Schmitz 2007) are complementary and hunting modes differ, as was the case in our system. Remarkably, the mortality rates measured within all three pairwise combinations of predators were almost perfectly intermediate between mortality within the pure-species treatments (Fig. 2). The alternative situation, in which multiple predators overlap extensively in habitat domain, applies in our study to the treatments in which conspecific predators foraged together. Overlap in microhabitat enables interference or intraguild predation/cannibalism among predators, and this in turn leads to reduced risk in multi-predator situations relative to the linear expectation (Sih et al. 1998; Schmitz 2007; Woodcock and Heard 2011). Interestingly, we observed a density-dependent reduction in risk only in Anax, the predator species in which individuals represent the most danger to one another. Cannibalism is well known in Notonecta, but not between adults (Fox 1975; Sih 1982). In larval odonates, individuals are often distracted or disturbed, if not eaten outright, by conspecifics (McPeek and Crowley 1987; Van Buskirk 1992). Taken together, these results support the hypothesis that habitat complementarity generates substitutability of multiple predators, whereas habitat overlap causes risk reduction (Schmitz 2007).

This conclusion implies that essential differences among predators in microhabitat or foraging mode were present even in the artificial environments of our mesocosms. Schmitz (2007) noted that simplistic experimental venues can enhance emergent MPEs by constraining habitat use such 
that predators interact more strongly than they would in nature. Artificial environments can cause overlap between habitat domains that would naturally be complementary. Indeed, there is evidence that multi-predator effects are sensitive to habitat structure (Siddon and Witman 2004; Warfe and Barmuta 2004). This was apparently not a problem in our study. Non-interactive effects under predator species combinations, and risk-reduction between conspecific odonates, suggest that the three predators were able to find zones within the tubs for foraging on vegetation (Anax), on the bottom (Triturus), and in open water (Notonecta).

Classic community theory begins with basic assumptions about the equivalence of individuals and density-independence of interaction strengths (Slobodkin 1955; Levins 1968; Vandermeer 1970). Although these assumptions are known to be violated in many instances, they applied reasonably well in our system. The three predator species that we studied could be treated as independent functional units in models that evaluate prey performance or community dynamics. Likewise, individuals within species could be represented as largely independent entities; the exception of Anax can be understood based on the biology of odonate larvae. Although this outcome is not typical of some other studies, our results are useful because they suggest that models of the effects of predators on multi-species systems may be able to treat their effects as independent when predators with non-overlapping functional attributes are considered.

Acknowledgments Many thanks to Ross Alford, Craig Osenberg, and Heinz-Ulrich Reyer for constructive comments on the manuscript, to Jasmin Winkler for help with the experiments, and to the Swiss National Science Foundation for financial support (31003A-113807).

\section{References}

Billick I, Case T (1994) Higher order interactions in ecological communities: what are they and how can they be detected? Ecology 75:1530-1543

Burnham PK, Anderson DR (2002) Model selection and multimodel inference. Springer, New York

Carey MP, Wahl DH (2010) Interactions of multiple predators with different foraging modes in an aquatic food web. Oecologia 162:443-452

Casula P, Wilby A, Matthew MB (2006) Understanding biodiversity effects on prey in multi-enemy systems. Ecol Lett 9:995-1004

Corbet PS (1957) The life-history of the emperor dragonfly Anax imperator Leach (Odonata, Aeshnidae). J Anim Ecol 26:1-69
Folsom TC, Collins NC (1984) The diet and foraging behavior of the larval dragonfly Anax junius (Aeshnidae), with an assessment of the role of refuges and prey activity. Oikos 42:105-113

Fox LR (1975) Factors influencing cannibalism, a mechanism of population limitation in predator Notonecta hoffmanni. Ecology 56:933-941

Griffen BD (2006) Detecting emergent effects of multiple predator species. Oecologia 148:702-709

Griffiths RA (1996) Newts and salamanders of Europe. Academic Press, London

Ives AR, Cardinale BJ, Snyder WE (2005) A synthesis of subdisciplines: predator-prey interactions, and biodiversity and ecosystem functioning. Ecol Lett 8:102-116

Jolliffe PA (2000) The replacement series. J Ecol 88:371-385

Levins R (1968) Evolution in changing environments. Princeton University Press, Princeton

Lima S (2002) Putting predators back into behavioural predator-prey interactions. Trends Ecol Evol 17:70-75

McPeek MA, Crowley PH (1987) The effects of density and relative size on the aggressive behaviour, movement and feeding of damselfly larvae (Odonata: Coenagrionidae). Anim Behav 35:1051-1061

Schmitz OJ (2007) Predator diversity and trophic interactions. Ecology 88:2415-2426

Siddon CE, Witman JD (2004) Behavioral indirect interactions: multiple predator effects and prey switching in the rocky subtidal. Ecology 85:2938-2945

Sih A (1982) Foraging strategies and the avoidance of predation by an aquatic insect, Notonecta hoffmanni. Ecology 63:786-796

Sih A, Crowley P, McPeek M, Petranka J, Strohmeier K (1985) Predation, competition, and prey communities: a review of field experiments. Annu Rev Ecol Syst 16:269-311

Sih A, Englund G, Wooster D (1998) Emergent impacts of multiple predators on prey. Trends Ecol Evol 13:350-355

Slobodkin LB (1955) Conditions for population equilibrium. Ecology 36:530-533

Streams FA (1987) Foraging behavior in a notonectid assemblage. Am Midl Nat 117:353-361

Van Buskirk J (1992) Competition, cannibalism, and size-class dominance in a dragonfly. Oikos 65:455-464

Van Buskirk J (2005) Local and landscape influence on amphibian occurrence and abundance. Ecology 86:1936-1947

Vance-Chalcraft HD, Soluk DA (2005) Multiple predator effects result in risk reduction for prey across multiple prey densities. Oecologia 144:472-480

Vance-Chalcraft HD, Soluk DA, Ozbum N (2004) Is prey predation risk influenced more by increasing predator density or predator species richness in stream enclosures? Oecologia 139:117-122

Vandermeer JH (1970) The community matrix and the number of species in a community. Am Nat 104:73-83

Warfe DM, Barmuta LA (2004) Habitat structural complexity mediates the foraging success of multiple predator species. Oecologia 139:171-178

Wilbur HM, Fauth JE (1990) Experimental aquatic food webs: interactions between two predators and two prey. Am Nat 135:176-204

Woodcock BA, Heard MS (2011) Disentangling the effects of predator hunting mode and habitat domain on the top-down control of insect herbivores. J Anim Ecol 80:495-503 